\title{
Analytical Optimization of Chassis Frame for 40ft Dual-Axle Flatbed Trailer Design
}

\author{
Ashif Iqubal1, S. M. Oak ${ }^{2}$ R. S.Kharatmal ${ }^{3}$ \\ ${ }^{I}$ Pursuing M.E. Mechanical Engineering, Department, Vishwakarma Institute of Technology, Pune, India \\ ${ }^{2}$ Professor, Mechanical Engineering, Department, Vishwakarma Institute of Technology, Pune, India \\ ${ }^{3}$ OTL, Pune, India
}

\begin{abstract}
This article will review a design and analysis study that reduces trailer chassis mass while minimizing the total cost impact. Design approaches, material selections and proposed section were reviewed. The Trailer chassis main member were quantified and summarized to create an overall mass and weighted cost estimate for a low mass Trailer.
\end{abstract}

Keywords: Optimization, Chassis frame, Flat bed trailer, Shear stress

\section{INTRODUCTION}

India Truck Industry, also known as Road Goods Transport Industry (RGTI) or Indian trucking industry, has played a major role in the Indian trade and commerce for the decades. Along with railways, Indian truck industry has also played an instrumental role in moving goods form one part of the country to another.

From 1950-51 to 1990-91, the truck industry in India has seen a growth rate of $7.2 \%$. Currently, there are more than 1300 trucks per million population, whereas the utilization of trucks is more than $70,000 \mathrm{kms}$ per annum.

The reason behind the success of India truck industry is the added advantage of road transport over the railways. Trucks can accept goods in small quantities, can reach rural and hill areas, and also require less time than the rail for loading and unloading of goods. As a result, India truck industry cemented its place in goods transportation. With passage of time, truck industry in India has involved a good number of Indian automobile giants including Tata Motors, Hindustan Motors, Ashok Leyland, Mahindra and Mahindra, Force Motors, Swaraj Maza, Eicher etc.

Due to increase in the fuel prices and all it is now necessary to make the trucking less costly. By making the trailers economic is one way of doing it. This project is the effort to do the same. By reducing the dimensions of the trailer parts such as beam the reduction of weight is possible which will consequently cause the reduction in the cost of trailer.

The trailer given is $40 \mathrm{ft}$ dual axle semi trailer. The objective is to reduce the weight of the trailer by 10 $\%$. By selecting appropriate size of beams the objective is completed.

\section{OBJECTIVE / Problem Statement}

The objectives of this paper are:

i) To reduce the weight of the trailer by $10 \%$. By selecting appropriate size of main member beams the objective is completed.

iii) The Trailer chassis main member and cross member were quantified and summarized to create an overall mass and weighted cost estimate for a low mass Trailer.

iv) To develop a new trailer chassis.

\section{ANALYTICAL APPROACH FOR OPTIMIZATION}

The first paragraph under each heading or subheading should be flush left, and subsequent paragraphs should have a five-space indentation.

\subsection{Design Input: 40ft Dual-Axle Flatbed Trailer Design \\ Load coming on each twist lock \\ Payload \\ Gross vehicle weight of trailer \\ Overall length of trailer \\ Overall width of trailer}




\subsection{Load Distribution On Kingpin And Rear Axle Centerline Chart}

\begin{tabular}{|l|l|l|l|l|}
\hline Sr.No. & Title & Weight $(\mathbf{k g})$ & $\begin{array}{l}\text { Load on Kingpin } \\
\left(\mathbf{R}_{\mathbf{k}}\right) \mathbf{k g}\end{array}$ & $\begin{array}{l}\text { Load on rear axle } \\
\text { centre line }\left(\mathbf{R}_{\mathbf{A}}\right) \mathbf{k g}\end{array}$ \\
\hline 1 & $\begin{array}{l}\text { Load on rear side } \\
(4.5 T \text { x 2) (Right side) }\end{array}$ & 9000 & - & 9000 \\
\hline 2 & $\begin{array}{l}\text { Load on front side } \\
(4.5 T \text { x }) \text { (Right side) }\end{array}$ & 9000 & 9000 & - \\
\hline 3 & Load on centre left (case1) & 9000 & 3520 & 5480 \\
\hline 4 & Load on centre right (case2) & 9000 & 3227 & 5773 \\
\hline 5 & Self weight of chassis frame & 4000 & 1643 & 2357 \\
\hline & & 40,000 & 17,390 & 22,610 \\
\hline
\end{tabular}

Table 1 - load distribution chart on kingpin and rear axle

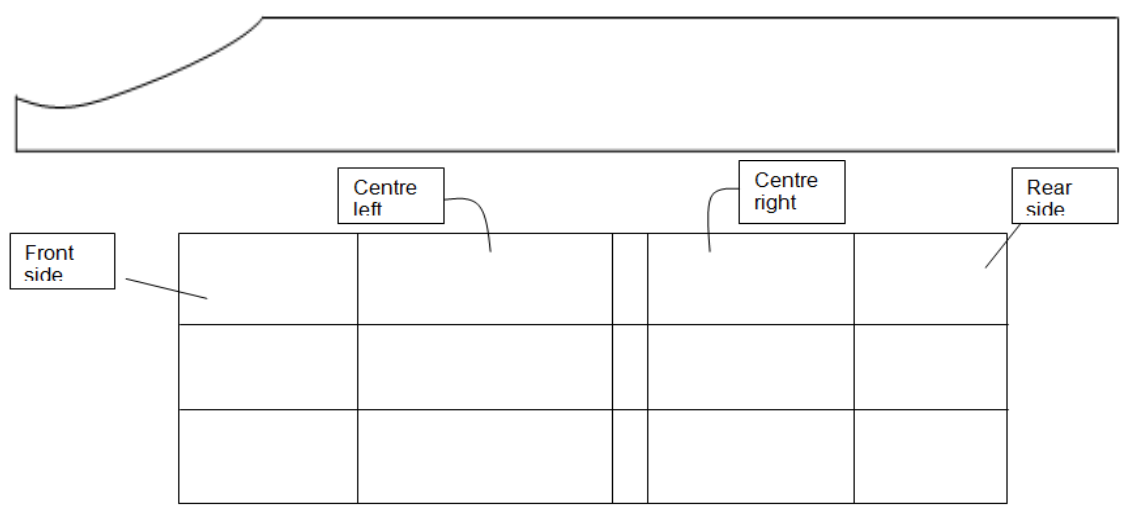

Fig1. Trailer front and top view

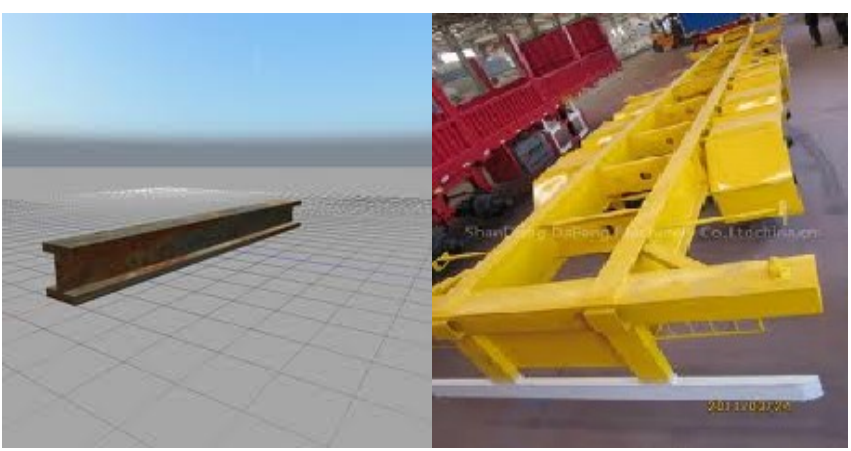

Fig.2: Main member design

\subsection{STUDY OF DIFFERENT PROPOSED SECTIONS OF COMPANIES WORLD OVER}

\begin{tabular}{|l|l|l|l|l|l|}
\hline Subject & $\begin{array}{l}\text { Existing } \\
\text { Section }\end{array}$ & \multicolumn{2}{l|}{ Proposed Sections } \\
\hline \multicolumn{2}{|l|}{} & I & II & III & IV \\
\hline Top Width (mm) & 152.9 & 150 & 140 & 146 & 140 \\
\hline Top Thickness (mm) & 13.3 & 12 & 14 & 14 & 14 \\
\hline Web Thickness mm) & 8.1 & 6 & 6 & 6 & 6 \\
\hline Bottom Width(mm) & 152.9 & 150 & 140 & 146 & 140 \\
\hline Bottom Thickness mm) & 13.3 & 12 & 16 & 16 & 16 \\
\hline Total Height (mm) & 454.6 & 500 & 500 & 500 & $\mathbf{7 3 . 2 0}$ \\
\hline Area $\left(\mathbf{c m}^{2}\right)$ & $\mathbf{7 6 . 2 3}$ & $\mathbf{6 4 . 5 6}$ & $\mathbf{7 0 . 2 0}$ & & $\mathbf{7 3 . 5 0}$ \\
\hline
\end{tabular}

Section selected - Section I with web of $6 \mathrm{~mm}$ Refrence

\begin{tabular}{|l|l|l|}
\hline \multirow{2}{*}{$\begin{array}{l}\text { Proposed } \\
\text { Sections }\end{array}$} & & CNHTC.Fujian special Vehicles co.Ltd. (made-in-china.com) \\
\cline { 2 - 3 } & $\bullet$ & Hubei Mingren Donfang Industry co.Ltd. (www.himfr.com) \\
\cline { 2 - 3 } & $\bullet$ & Wall cargo semitrailer (factory.dhgate.com) \\
\hline
\end{tabular}




\subsection{Section Selection}

\begin{tabular}{|l|l|l|}
\hline Subject & Existing Section \\
\hline Figure & \\
\end{tabular}

\subsection{Calculations Of Proposed Section:}

\begin{tabular}{|l|l|l|}
\hline Area & $=$ & {$\left[\left(2 \mathrm{~b} \times \mathrm{t}_{\mathrm{f}}\right) \times 2+\left(\mathrm{d}_{1} \times \mathrm{t}_{\mathrm{w}}\right)\right]$} \\
& $=$ & {$[(150 \times 12) \times 2+(476 \times 6)]$} \\
& $=$ & $3600+3808$ \\
& $=$ & $6456 \mathrm{~mm}^{2}$ \\
& $=$ & $64.56 \mathrm{~cm}^{2}$ \\
\hline Moment Of Inertia $\left(\mathrm{I}_{\mathrm{xx}}\right)$ & $=$ & {$\left[(1 / 12) \times 2 \mathrm{~b} \times \mathrm{d}^{3}\right]-\left[(1 / 12) \times\left(2 \mathrm{~b}-\mathrm{t}_{\mathrm{w}}\right) \mathrm{xd}_{1}{ }^{3}\right]$} \\
& $=$ & {$\left[(1 / 12) \times 150 \times 500^{3}\right]-\left[(1 / 12) \times(150-6) \times 476^{3}\right]$} \\
& $=$ & $156250 \times 10^{4}-12942 \times 10^{4}$ \\
& $=$ & $26830 \times 10^{4} \mathrm{~mm}^{4}$ \\
\hline Section Modulus $\left(\mathrm{z}_{\mathrm{xx}}\right)$ & $=$ & $\left.\left.\left[(1 / 6) \times \mathrm{b}^{3} \times \mathrm{d}^{3} / \mathrm{d}\right)\right]-\left[(1 / 6) \times 2 \mathrm{~b}_{1} \times \mathrm{x}_{1}{ }^{3} / \mathrm{d}\right)\right]$ \\
& $=$ & {$\left[(1 / 6) \times 2 \mathrm{~b} \times \mathrm{d}^{2}\right]-\left[(1 / 6) \times 2 \mathrm{~b}_{1} \times\left(\mathrm{d}_{1}{ }^{3} / \mathrm{d}\right)\right]$} \\
& $=$ & {$\left[(1 / 6) \times 150 \times 500^{2}\right]-\left[(1 / 6) \times 144 \times\left(456^{3} / 500\right)\right]$} \\
& $=$ & $6250 \times 10^{3}-5176.80 \times 10^{3}$ \\
& $=$ & $1073.2 \times 10^{3} \mathrm{~mm}^{3}$ \\
& $=$ & $1073.2 \mathrm{~cm}^{3}$ \\
& &
\end{tabular}

\section{Calculations Of Shear Stresses}

\section{Maximum shear stress}

Maximum intensity of Shear stress will occur at the neutral axis. The shear stress is given by,

Where,

$$
\tau=\frac{s . a y}{I x x * t w}
$$

$$
\begin{aligned}
& \mathrm{S}=\text { Maximum shear force, } \mathrm{kg} . \\
& \mathrm{ay}=\text { Moment of the area above neutral axis, about the neutral axis. } \\
& \mathrm{I}_{\mathrm{xx}}=\text { Moment of inertia, } \mathrm{mm}^{4} \text {. } \\
& \mathrm{t}_{\mathrm{w}}=\text { Breadth of web, } \mathrm{mm} \text {. }
\end{aligned}
$$

(Moment of the area above neutral axis $=150 \times 12 \times 244+6 \times 238 \times 119$

And about the neutral axis)

$$
\begin{gathered}
=60.91 \times 10^{4} \mathrm{~mm}^{3} \\
\text { Maximum shear stress } \tau_{\max }=\left(\begin{array}{c}
\left.900 \times 60.91 \times 10^{4}\right) /\left(26830 \times 10^{4} \times 6\right) \\
=3.40 \mathrm{~kg} / \mathrm{mm}^{2}
\end{array}\right.
\end{gathered}
$$




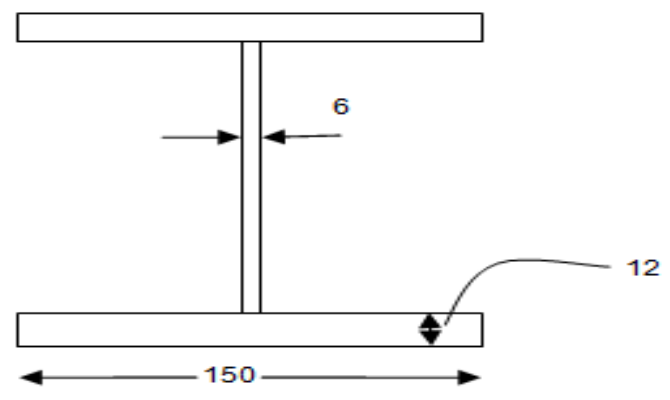

Fig.3: Beam with dimensions

Shear stress just above the junction of the flange and web

(Shear stress in the Flange in the web at $238 \mathrm{~mm}$ from neutral axis)

$$
\begin{aligned}
\tau & =\frac{\text { s.ay }}{I x x * 2 b} \\
& =9000 \times\{150 \times 12 \times[(12 / 2)+238]\} /\left(26830 \times 10^{4} \times 150\right) \\
& =0.09 \mathrm{~kg} / \mathrm{mm}^{2}
\end{aligned}
$$

Shear stress just below the junction of the flange and web

(Shear stress in the Web at $238 \mathrm{~mm}$ from neutral axis)

$$
\begin{aligned}
\tau & =\frac{\text { s.ay }}{I x x * t w}=9000 \times\{150 \times 12 \times[(12 / 2)+238]\} /\left(26830 \times 10^{4} \times 150\right) \\
& =2.25 \mathrm{~kg} / \mathrm{mm}^{2}
\end{aligned}
$$
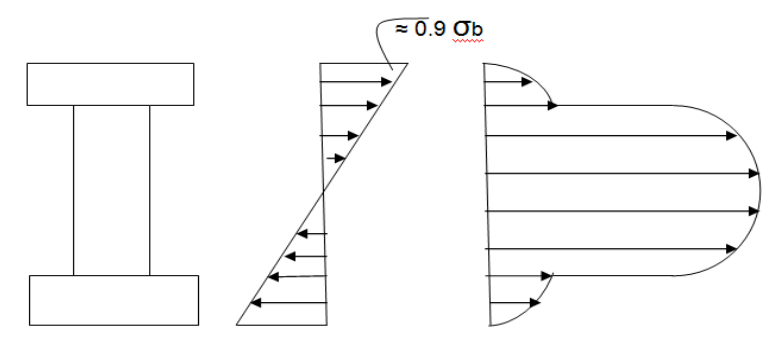

Fig.4 Shear and Bending Stress Distribution diagram

\section{$\%$ Reduction in Area}

$=$ Area of existing section - Area of proposed section / area of existing section

$=[76.23-64.56 / 76.23] * 100$

$=15 \%$

\section{Weld Size Calculations At The Junction Of Flange Plate And Web Plate} 4.1 Input Data:

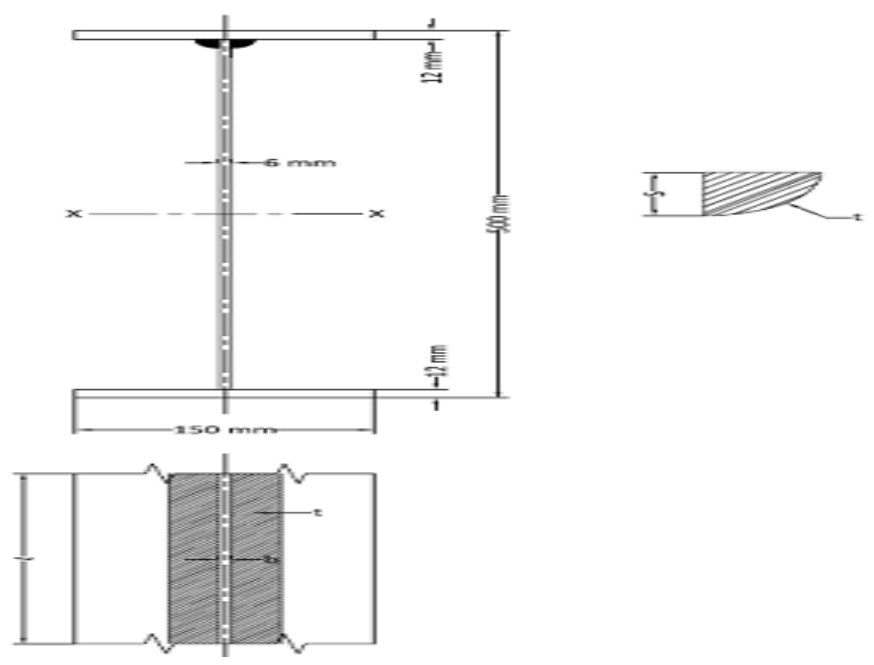




\begin{tabular}{|l|l|l|}
\hline Maximum bending moment $(\mathrm{M})$ & $=$ & $148 \times 10^{5} \mathrm{~kg}-\mathrm{mm}$. (from fig.2) \\
\hline Web thickness (b) & $=$ & $6 \mathrm{~mm}$. \\
\hline Length of the weld (1) & $=$ & $12135 \mathrm{~mm}$. (from fig.1) \\
\hline Allowable bending stress in weld $(\sigma \mathrm{b})$ & $=$ & $15 \mathrm{~kg} / \mathrm{mm}^{2}$ \\
\hline
\end{tabular}

Case I: Weld material of allowable bending stress $15 \mathrm{~kg} / \mathrm{mm}^{2}$

i) Required Section Modulus:

Formula:

$\mathrm{Z}_{\mathrm{R}}=\mathrm{M}_{\mathrm{w}} / \boldsymbol{\sigma b}$ [Ref. Eqn. (2.5)-Strength of materials by Nirali Publication]

Where,

$$
\begin{aligned}
& \mathrm{M}_{\mathrm{w}}=\text { Bending Moment at the welding section, kg-mm } \\
& =\text { Maximum Bending moment x } 0.9 \text { (from fig.5) } \\
& =148.26 \times 10^{5} \times 0.9 \\
& =133.43 \times 10^{5} \mathrm{~kg}-\mathrm{mm} \\
& \therefore \quad \mathrm{Z}_{\mathrm{R}}=\mathrm{M}_{\mathrm{w}} / \sigma \mathrm{b} \\
& =\left(133.43 \times 10^{5}\right) / 15 \\
& =8.89 \times 10^{5} \mathrm{~mm}^{3}
\end{aligned}
$$

$\therefore$ Required Section Modulus is $8.89 \times 10^{5} \mathrm{~mm}^{3}$

ii) Available Section Modulus:

$$
\text { Formula: }
$$

$$
\begin{aligned}
\mathrm{Z}_{\mathrm{A}} & =\mathrm{t} \times \mathrm{b} \times 1 \\
\mathrm{Z}_{\mathrm{A}} & =\mathrm{t} \times 6 \times 12135 \\
& =72810 \mathrm{t}
\end{aligned}
$$

iii) Weld leg calculation:

For weld leg calculation we put following condition,

Available Section Modulus = Required Section of Modulus

$$
\left(Z_{\mathrm{A}}\right)=\left(Z_{\mathrm{R}}\right)
$$

$$
\begin{aligned}
72810 \times \mathrm{t} & =8.89 \times 10^{5} \\
\mathrm{t} & =12.20 \mathrm{~mm} \approx 13 \mathrm{~mm}
\end{aligned}
$$

$\therefore$ Weld throat $(\mathrm{t})=$ weld leg $(\mathrm{s})=13 \mathrm{~mm} \quad$..... (From input data)

The weld leg is $\underline{13 \mathrm{~mm}}$ for allowable bending stress of $\underline{15 \mathrm{~kg} / \mathrm{mm}^{2}}$ of the weld

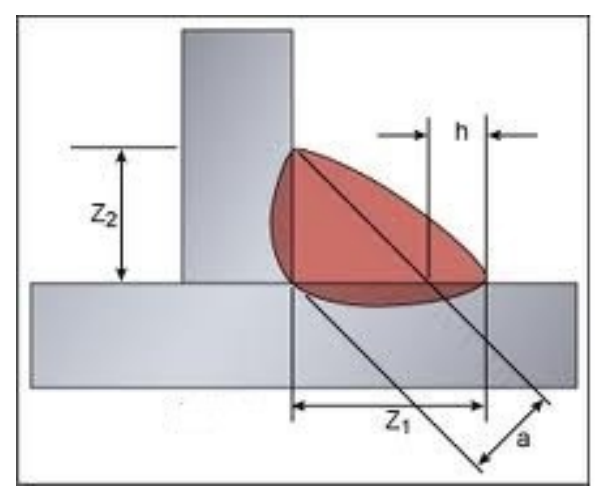

Case II: Weld material of allowable bending stress $16 \mathrm{~kg} / \mathrm{mm}^{2}$

i) Required Section Modulus:

$$
\text { Formula: }
$$

$$
\mathrm{Z}_{\mathrm{R}}=\mathrm{M}_{\mathrm{w}} / \sigma \mathrm{b}
$$

Where,

$$
\begin{aligned}
& \mathrm{M}_{\mathrm{w}}=\text { Bending Moment at the welding section, } \mathrm{kg}-\mathrm{mm} \\
& =\text { Maximum Bending moment x } 0.9 \text { (from fig 5) } \\
& =148.26 \times 10^{5} \times 0.9 \\
& =133.43 \times 10^{5} \mathrm{~kg}-\mathrm{mm} \\
& \therefore \quad \mathrm{Z}_{\mathrm{R}}=\mathrm{M}_{\mathrm{w}} / \boldsymbol{\sigma b} \\
& =\left(133.43 \times 10^{5}\right) / 16 \\
& =8.33 \times 10^{5} \mathrm{~mm}^{3}
\end{aligned}
$$

$\therefore$ Required Section Modulus is $8.33 \times 10^{5} \mathrm{~mm}^{3}$

ii) Available Section Modulus:

Formula:

$\mathrm{Z}_{\mathrm{A}}=\mathrm{t} \times \mathrm{b} \times 1$ 


$$
\begin{aligned}
Z_{\mathrm{A}} & =\mathrm{t} \times 6 \times 12135 \\
& =(72810 \times \mathrm{t}) \mathrm{mm}^{3}
\end{aligned}
$$

iii) Weld leg calculation:,

For weld leg calculation we put following condition,

Available Section Modulus = Required Section of Modulus

$$
\left(Z_{\mathrm{A}}\right)=\left(\mathrm{Z}_{\mathrm{R}}\right)
$$

$72810 \times \mathrm{t}=8.33 \times 10^{5}$

$$
\mathrm{t}=11.45 \mathrm{~mm} \approx 12 \mathrm{~mm}
$$

$\therefore$ Weld throat $(\mathrm{t})=$ weld leg $(\mathrm{s})=12 \mathrm{~mm} \quad \ldots$ (From input data)

The value of weld leg is $12 \mathrm{~mm}$ for allowable bending stress of $16 \mathrm{~kg} / \mathrm{mm}^{2}$ of the weld

4. EXISTING MIDDLE AND SIDE CROSS MEMBERS DESIGN
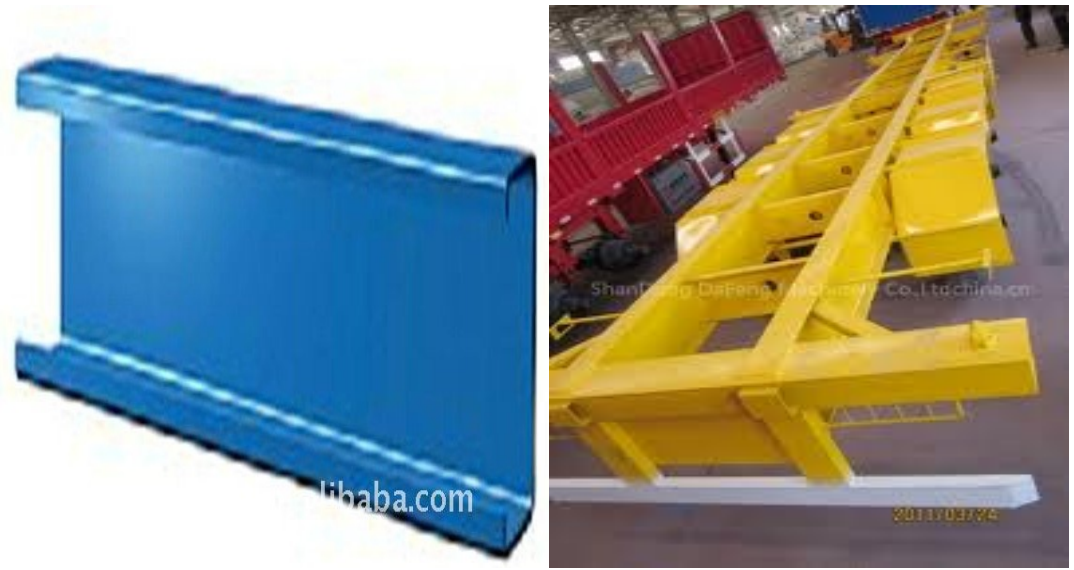

A) Input Data:

Fig.7 Cross Member

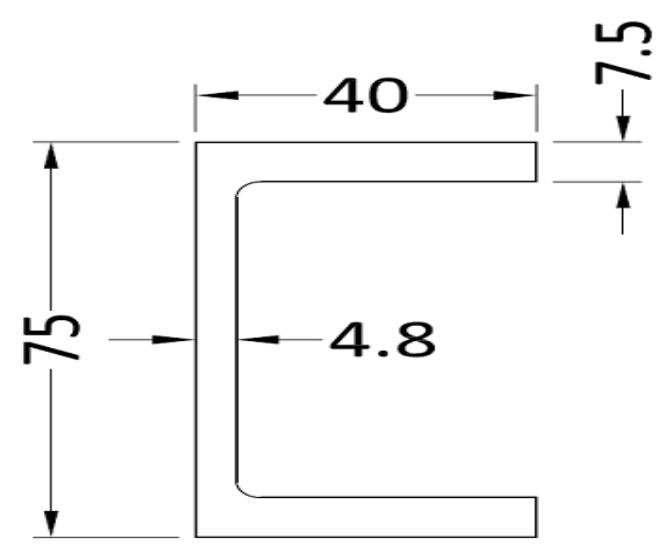

Fig. 8 existing cross member

Section: ISMC $-75 \times 40 \times 4.8$ thk x $7.14 \mathrm{~kg} / \mathrm{m}$

Weight per unit length

Moment of inertia $\left(\mathrm{I}_{\mathrm{xx}}\right)$

Section of modulus $\left(Z_{\mathrm{xx}}\right)$

Area of section

Load on Member

$$
\begin{aligned}
& =7.14 \mathrm{~kg} / \mathrm{m} \\
& =78.5 \mathrm{~cm}^{4} \\
& =16.14 \mathrm{~cm}^{3} \text { calculated in calculations } \\
& =9.1 \mathrm{~cm}^{2} \\
& =1166 \mathrm{~kg} \quad \text { Given }
\end{aligned}
$$

\subsection{Calculations Of Existing Middle And Side Cross Member}

1) Shear stress on beam

Shear stress on beam $(\mathbf{\tau})=(\mathrm{w} \times \mathrm{A}) / 2$

$$
\begin{array}{r}
\tau=1166 / 2 \times 9.1 \times 100 \\
=0.64 \mathrm{~kg} / \mathrm{mm}^{2}
\end{array}
$$

2) Bending moment

Bending moment $(\mathrm{M})=\mathrm{w} \times 1 / 12$

$$
=1166 \times 950 / 12
$$




$$
\begin{aligned}
& =92308.33 \mathrm{~kg}-\mathrm{mm} \\
& =92.3 \mathrm{~kg}-\mathrm{m}
\end{aligned}
$$

3) Section of Modulus

Section of modulus $\left(\mathrm{z}_{\mathrm{xx}}\right)=\mathrm{I}_{\mathrm{xx}} /(\mathrm{H} / 2)$

$$
\begin{aligned}
& =78.5 / 3.75 \\
& =20.96 \mathrm{~cm}^{3} \\
& =20966.66 \mathrm{~mm}^{3}
\end{aligned}
$$

4) Bending stress

$$
\begin{aligned}
\text { Bending stress } \boldsymbol{\sigma} \mathrm{b}= & \text { Bending moment }(\mathrm{M}) / \text { Section of modulus }\left(\mathrm{z}_{\mathrm{xx}}\right) \\
& =92308.33 / 20966.66 \\
& =4.4 \mathrm{~kg} / \mathrm{mm}^{2}
\end{aligned}
$$

4.2 PROPOSED MIDDLE AND SIDE CROSS MEMBER CALCULATIONS Input data:

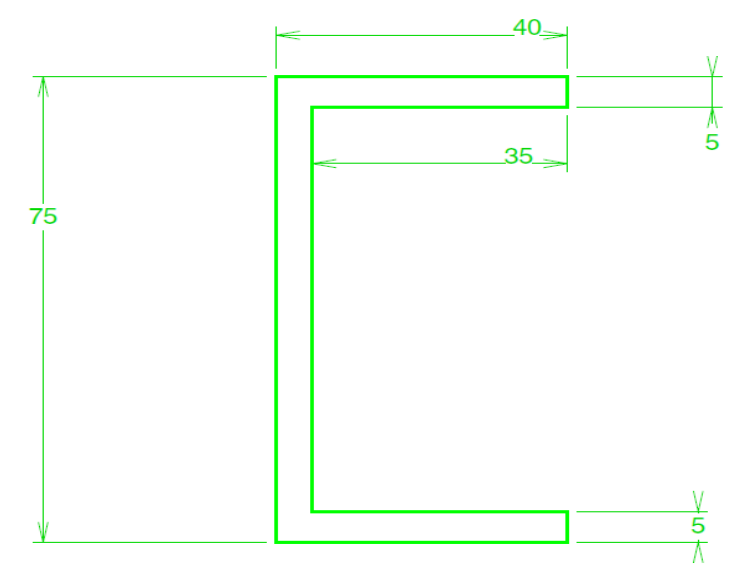

Fig.9 Proposed cross member

Section: $I S M C-75 \times 40 \times 5$ thk $x 6.08 \mathrm{~kg} / \mathrm{m}$

Weight per unit length

Moment of inertia $\left(\mathrm{I}_{\mathrm{xx}}\right)$

Section of modulus $\left(Z_{x x}\right)$

Area of section

\section{1) Area}

$$
\begin{gathered}
=6.08 \mathrm{~kg} / \mathrm{m} \\
=60.52 \mathrm{~cm}^{4} \\
=16.14 \mathrm{~cm}^{3} \\
=7.75 \mathrm{~cm}^{2}
\end{gathered}
$$

\section{Calculations}

$$
\begin{aligned}
& =775 \mathrm{~mm}^{2} \\
& =7.75 \mathrm{~cm}^{2}
\end{aligned}
$$

\section{2) Weight per unit length:}

For area $9.1 \mathrm{~cm}^{2}$

For area $7.75 \mathrm{~cm}^{2}$

weight $/$ unit length is $=7.14 \mathrm{~kg} / \mathrm{m}$

weight $/$ unit length $=$ ?

weight / unit length $\quad=7.75 \times 7.4 / 9.1$

for selected section

3) \% Reduction in load:

$$
=6.08 \mathrm{~kg} / \mathrm{m}
$$

$=$ weight per unit length of (existing section - proposed section)

Weight per unit length of existing section

$$
\begin{aligned}
& =7.14-6.08 / 7.14 \\
& =14.8 \%
\end{aligned}
$$

\section{4) Bending moment}

$$
\begin{aligned}
\text { Bending moment }(\mathrm{M}) & =\mathrm{w} \times 1 / 12 \\
& =1166 \times 950 / 12 \\
& =92308.33 \mathrm{~kg}-\mathrm{mm}
\end{aligned}
$$


5) Section of Modulus

Section of modulus $\left(\mathrm{z}_{\mathrm{xx}}\right)=\left(\mathrm{BH}^{2} / 6\right)-\left(\mathrm{bh}^{3} / 6 \mathrm{H}\right)$

$$
\begin{aligned}
& =\left(40 \times 75^{2} / 6\right)-\left(35 \times 65^{3} / 6 \times 75\right) \\
& =16140 \mathrm{~mm}^{3}
\end{aligned}
$$

\section{6) Moment of Inertia}

Moment of inertia $\mathrm{I}_{\mathrm{xx}}=$ Section of modulus $\mathrm{x} \mathrm{H} / 2$

$$
\begin{aligned}
& =16140 \times 75 / 2 \\
& =605250 \mathrm{~mm}^{4}
\end{aligned}
$$

\section{7) Bending stress}

Bending stress $(\sigma \mathrm{b})=$ Bending moment $(\mathrm{M}) /$ Section of modulus $\left(\mathrm{z}_{\mathrm{xx}}\right)$

$$
\begin{aligned}
& =92308.33 / 16140 \\
& =5.7 \mathrm{~kg} / \mathrm{mm}^{2}
\end{aligned}
$$

1. SUMMARY:

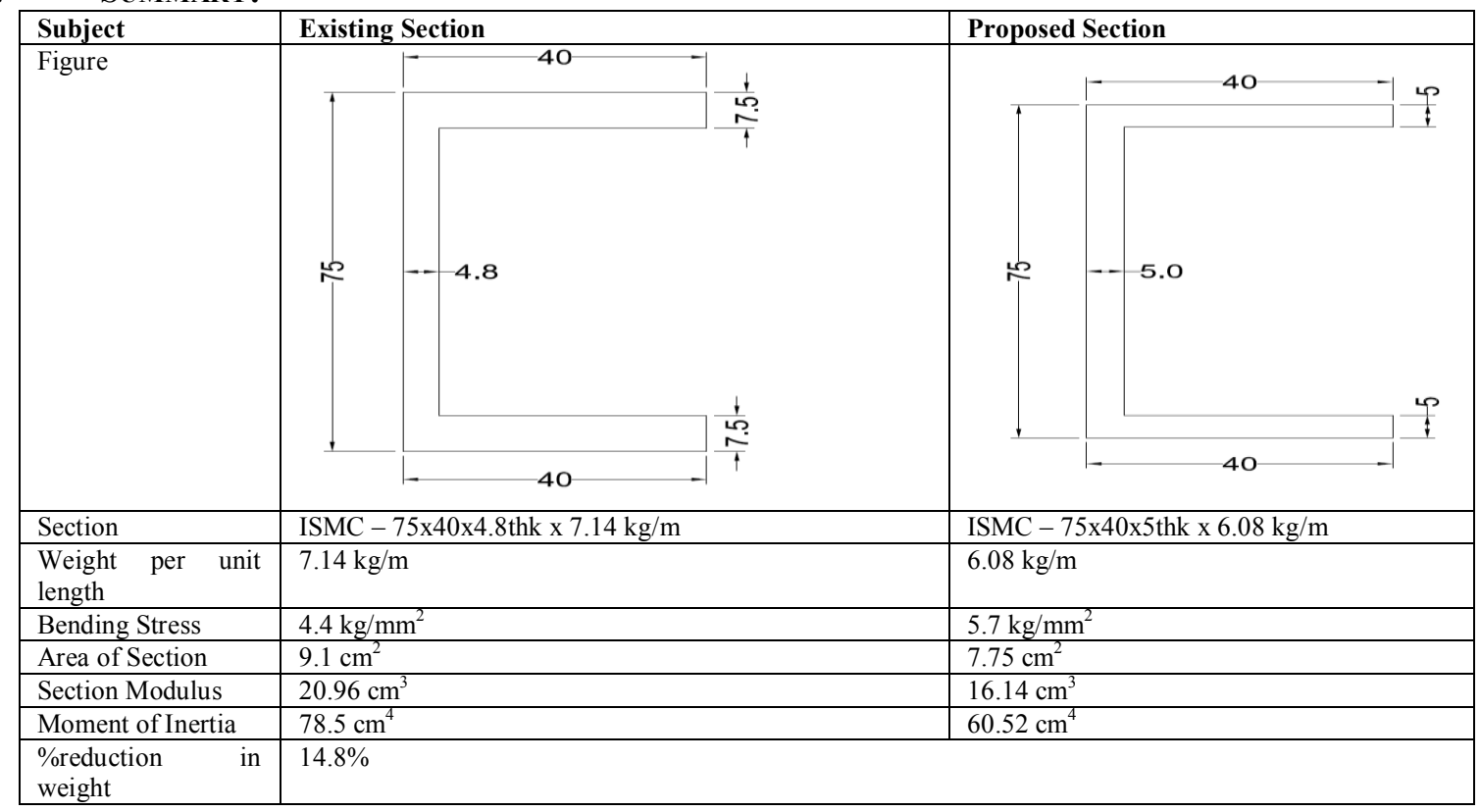

\section{CONCLUSION}

1. A conclusion for main member by modifying the size of the existing section the weight is reduces up to 15 $\%$.

2. The weld leg size for main member

Table -5 Weld Leg Size

\begin{tabular}{|l|ll|l|l|}
\hline Case & $\begin{array}{c}\text { Allowable bending stress in weld } \\
\left(\mathrm{kg} / \mathrm{mm}^{2}\right)\end{array}$ & Required section modulus $\left(\mathrm{mm}^{3}\right)$ & Weld leg $(\mathrm{mm})$ \\
\hline I & 15 & & $8.89 \times 10^{5}$ & 13 \\
\hline II & 16 & & $8.33 \times 10^{5}$ & 12 \\
\hline
\end{tabular}

3. For middle and side cross members by modifying the existing section the weight is reduced by $14.8 \%$.

4. The weight of the trailer is reduced to make it economical.

\section{Acknowledgements}

I would like to express my very great appreciation to Professor S.M Oak for his valuable and constructive suggestions during the planning and development of this research work. His willingness to give his time so generously has been very much appreciated.

I would also like to thank the Mr.G.Singhal, Managing Director of Pragya Technologies (India) Pvt.Ltd. 


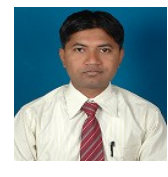

Pursuing M.E. (Design Engineering) from Vishwakarma Institute of Technology,

Pune, of University of Pune, having 3.10 years of

Industrial experience.

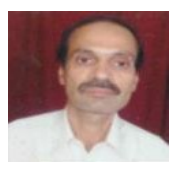

Assistant Professor at Vishwakarma Institute of Technology, Pune, India.

Received M.E. in Mechanical Engineering with Heat Power as specialization from College of Engineering, Pune..

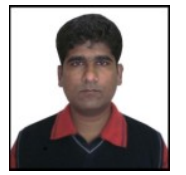

Pursuing M.E. (Design Engineering ) from Vishwakarma Institute of Technology,

Pune, of University of Pune, having 2.8 year of

industrial experience.

\section{REFERENCES}

\section{Journal Papers:}

[1] Murali M.R. Krisna, "Chassis Cross-Member Design Using Shape Optimization", Internasional Congress and Exposition Detroit, Michigan. February 23-26,1998.

[2] Kazuhiro Izu, Shinji Nishiwaki, Masataka Yoshimura. An Optimal Cross sectional Design Method. SAE 2003-01-2782, Page no 559 to 564

[3] A. D. M. Chauhan, B. Prof. S. B. Soni and C. Prof. A. M. Gohil. "Parametric Optimization of Hydraulic Modular Trailer Frame using ANSYS (APDL)". Institute of technology, nirma university, ahmedabad - 382481.

[4] Vijaykumar V. and Prof. R. I. Patel "Structural Analysis of Automotive Chassis Frame and Design Modification for Weight Reduction". International Journal of Engineering Research \& Technology (IJERT). 2012.

[5] Jeffrey W. Herrmann. "Evaluating Design optimization Models". ISR Technical report 2007-11.

[6] Fui T.H. and Rahman R.Abd. "Static and dynamics structural analysis of a 4.5 ton truck chassis". Jurnal Mekanikal, No. 24, pa ges Books:

[7] John Fenton (1996) Hand Book of Vehicle Design Analysis. MEP Ltd, UK.

[8] J.William Fitch, "Motor Truck Engineering Handbook-Fourth Edition", SAE Inc., Warrendale, U.S.A, 1993.

[9] Harbans S.R, 1962.The automobile, India, Scand \& Company Ltd. Page no: 37 to 48

[10] Prof.Dip-Ing.Jornsen R\& helmut.S, The automotive Chassis Engineering Principles. , A member of the hodder headline group. Page no 358 to 366

[11] John Robertson, An Introduction to Modern Vehicle Design. Page no 125 to 142.

[12] From Design Data Book --- PSG Tech 5.130

\section{Theses:}

[13] Ashif iqubal, Study Of Static And Dynamic Behaviour Of Trailer Chassis Frame, M.E dessertation., Vishwakarma Institute of Technology, Pune,India, 2013. 